\title{
Study on Non-Uniqueness of Taylor Vortex Flow Changing Inner Cylinder Acceleration Time
}

\author{
Hiroyuki Furukawa, Tomohito Suzuki \\ Department of Mechanical Engineering, Meijo University, Nagoya, Japan \\ Email: furukawa@meijo-u.ac.jp
}

How to cite this paper: Furukawa, H. and Suzuki, T. (2018) Study on Non-Uniqueness of Taylor Vortex Flow Changing Inner Cylinder Acceleration Time. World Journal of Mechanics, 8, 301-310. https://doi.org/10.4236/wjm.2018.88023

Received: July 25, 2018

Accepted: August 13, 2018

Published: August 16, 2018

Copyright $\odot 2018$ by authors and Scientific Research Publishing Inc. This work is licensed under the Creative Commons Attribution International License (CC BY 4.0).

http://creativecommons.org/licenses/by/4.0/ (c) (i) Open Access

\begin{abstract}
In concentrically rotating double cylinders consisting of a stationary outer cylinder a rotating inner cylinder, Taylor vortex flow appears. Taylor vortex flow occurs in journal bearings, various fluid machineries, containers for chemical reaction, and other rotating components. Therefore, the analysis of the flow structure of Taylor vortex flow is highly effective for its control. The main parameters that determine the modes of Taylor vortex flow of a finite length are the aspect ratio, Reynolds number Re. Aspect ratio is defined as the ratio of the cylinder length to the gap length between cylinders, and $R e$ is determined on the basis of the angular speed of the inner cylinder. Aspect ratio was set to be 4.0, and $R e$ to be values in the range from 100 to 1000 at intervals of 100. Thus far, a large number of studies on Taylor vortex flow have been carried out; however, the effects of the differences in initial conditions have not yet been sufficiently clarified. In this study, we changed the acceleration time of the inner cylinder in a numerical analysis, and examined the resulting changes in the mode formation and bifurcation processes. The acceleration time was changed from $1.0 \mathrm{~s}$ to $10.0 \mathrm{~s}$. As a result, a difference was observed in the final mode depending on the difference in the acceleration time. From this finding, non-uniqueness, which is a major characteristic of Taylor vortex flow, was confirmed. However, no regularities regarding the difference in mode formation were found and the tendency of the mode formation process was not specified. Moreover, the processes of developing the vortex resulting in different final modes were monitored over time by visual observation. Similar flow behaviors were initially observed after the start of the calculation. Then, a bifurcation point, at which the flow changed to a mode depending on the acceleration time observed, and finally the flow became steady. In addition, there was also a difference in the time taken for the well-developed flow to reach the steady state. Both EFD (Experimental Fluid Dynamics) and CFD (Computational Fluid Dynamics) results show good agreement qualitatively.
\end{abstract}




\section{Keywords}

Rotating Cylinders, Flow Instability, Bifurcation, EFD and CFD, Non-Uniqueness

\section{Introduction}

Since the classical research by G. I. Taylor in 1923 [1], the Taylor vortex has been widely investigated and even limited to major studies, more than 1500 research papers have been published so far. The Taylor vortex is regarded as one of the most important vortexes in "flow". In engineering, many studies have been conducted in relation to fluid bearings (bearings, spindle motors, and journal bearings) and welding methods.

Let us briefly explain the Taylor vortex. When fluid flows in a narrow space between inner and outer cylinders of two coaxial rotating cylinders sharing the central axis, the fluid flow in the circumferential direction of the cylinder with the rotation of one of the cylinders or both cylinders. Thereby, if the centrifugal force working on the fluid and surrounding pressure are imbalanced, the vortex rotating alternatively in the opposite direction having an axis in the direction of flow is formed. This vortex is called "Taylor vortex".

When the main velocity of the inner cylinder is gradually increased from zero, a Couette flow first appears in the space between two rotating cylinders. If the velocity is further increased, the flow changes to Taylor vortex flow in which a torus-like flow called a cell overlaps, wave Taylor vortex flow, and turbulent flow several times. Since the Taylor vortex flow is observed in journal bearings, various fluid machines, and chemical reaction vessels, it is technologically important to elucidate its mechanism. Especially, the prediction and control of the Taylor vortex flow is important because the changes in its mode induce the unsteady changes in torque and reaction rate [2] [3] [4] [5].

It is known that one of the characteristics of the Taylor vortex is its non-uniqueness. Namely, even at the same aspect ratio and same Reynolds number, the final mode, which is the finally stabilized mode of flow, is sometimes different due to the changes in the acceleration time of the inner cylinder. Benjamin and Mullin [6] experimentally showed that there are multiple different modes including anomalous mode at the same aspect ratio and the same Reynolds number. Alziary de Roquefort and Crillaud [7] demonstrated that multiple stable modes appear at the same aspect ratio and the same Reynolds number based on the numerical analysis of the steady axisymmetric Navier-Stokes equation. Furukawa, et al. [8] changed the initial flow field between the inner and outer cylinders in a numerical analysis, and examined the resulting changes in the mode formation and bifurcation processes. Furukawa, et al. [9] built a three-dimensional visualization system to analyze the non-unique mode formation process. The proposed system can help to investigate the relationship be- 
tween the mode bifurcation process and the volume-averaged energy.

However, the previous studies have been limited to the elucidation of the non-uniqueness of the Taylor vortex only by experiments or only by numerical calculations. There has been no research that compares the experimental results and numerical calculations. The objective of the present study is to elucidate more deeply the non-uniqueness of the Taylor vortex by comparing experimental results and calculations.

\section{Experiment and Numerical Method}

\subsection{Experiment Device}

Figure 1 shows a schematic of Taylor vortex flow investigated in this study. The radii of the inner and outer cylinders are $r_{\text {in }}=20 \mathrm{~mm}$ and $r_{\text {out }}=30 \mathrm{~mm}$, respectively, and the gap between the cylinders is $r_{\text {out }}-r_{\text {in }}=10 \mathrm{~mm}$. The inner cylinder is rotated at an angular velocity of $\omega$. A pulley is attached to a belt wheel below the center of the cylinder to rotate the inner cylinder. Here, only the inner cylinder is rotated because the outer cylinder is fixed. A slide ring is attached to the upper ends of the cylinders to fix their positions and to change the aspect ratio of the gap, which is used as the observation target. The setup also includes a servo motor, a servo amplifier (MR-J2-A, Mitsubishi Electric Corporation), a servo motor control unit (Tateiwa Shoji), and aNd-YAG laser device (1.5 W, DSPP Green Laser, Japan Laser Corporation).

A mixture of distilled water and glycerin with a mixing ratio of $8: 2$ is used as the fluid specimen in this study. The specific gravity of glycerin is 1.26 . The advantages of using glycerin are that 1) the Reynolds number can be controlled by varying its viscosity and 2) it helps tracer particles float in the fluid specimen. The tracer particles used in this study are fine fluorescent particles with a high luminescence, which were added to the fluid to visualize flows. FLUOSTAR (EBM Corporation) particles are used in this study. When laser light is irradiated onto these particles perpendicularly to the observation direction, flows can be observed owing to the reflection of light.

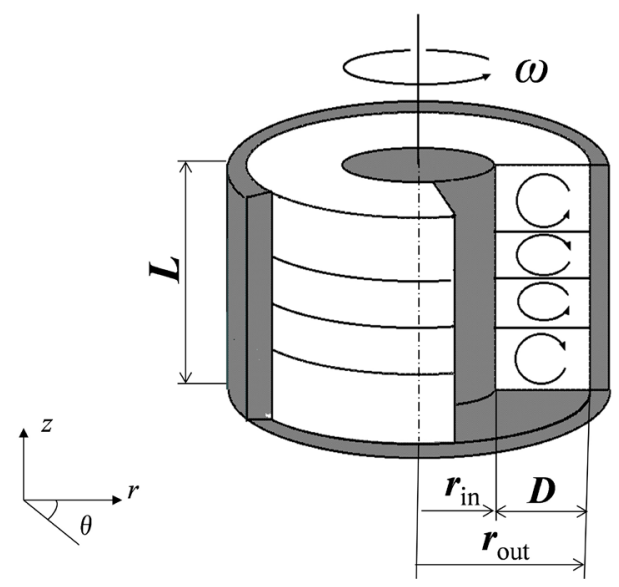

Figure 1. Schematic of Taylor vortex flow. 
The aspect ratio was determined, and the rotation speed of the servo motor required to give the desired Reynolds number and the time required to reach this value (i.e., the rising time) were input to the servo amplifier. Next, the motor was driven and photographs were taken using a high-speed video camera (VCC-H1000, Digimo Co., Ltd.) when the vortex stabilized to form the final-mode flow. The parameters that govern Taylor vortex flow are the Reynolds number and aspect ratio, as mentioned above. The Reynolds number $R e$ is a dimensionless number, defined as the ratio of the inertial force in the equation of motion to the viscous force, and is given by

$$
R e=\frac{V D}{v}
$$

here, $V$ is a representative velocity, given as the rotation speed of the inner cylinder $\left(r_{\text {in }} \omega\right), D$ is the width of the gap between the inner and outer cylinders, given by the difference between their representative radii as $r_{\text {out }}-r_{\text {in }}$, and $v$ is the kinematic viscosity of the fluid. The aspect ratio $\Gamma$ is defined as the ratio of the cylinder length $L$ to the gap width $r_{\text {out }}-r_{\text {in }}$, and is given by

$$
\Gamma=L / D
$$

Various final modes of Taylor vortex flow are formed by changing these parameters. In this study, we define a flow in which the relative time change in the torque applied to the cylinder wall is less than $10^{-6}$ as a steady flow. The Stokes flow function (Equation (3)) is used to visualize the flow.

$$
u=-\frac{1}{r} \frac{\partial \psi}{\partial z} \quad w=\frac{1}{r} \frac{\partial \psi}{\partial r}
$$

\subsection{Numerical Method}

The governing equations are the axisymmetric unsteady incompressible NavierStokes equation with cylindrical coordinates $(r, \theta, z)$ (Equation (4)) and the equation of continuity (Equation (5)).

$$
\begin{gathered}
\frac{\partial u}{\partial t}+(u \cdot \nabla) u=-\nabla p+\frac{1}{\operatorname{Re}} \nabla^{2} u \\
\nabla \cdot u=0
\end{gathered}
$$

here, $u$ is a velocity vector with the components of $(u, v, w), t$ is a dimensionless time based on a representative time, and $p$ is the pressure. The discretization of the governing equations is based on the MAC method. For time integration, Euler's explicit method is used. For space integration, the QUICK method is used for the convection terms, and the secondary central difference method is used for other terms. As an initial condition, the velocity is set to 0 in all regions. As boundary conditions, the no-slip condition at each cylinder wall is applied for the velocity, and the Neumann condition based on the equation of motion is applied for the pressure. Staggered grids are used as calculation grids and are assumed to have regular intervals in each direction. 


\section{Results}

\subsection{Experimental Results}

In the present study, we observed the vortex flow that reached steady state by changing the acceleration time and Reynolds number under four conditions, i.e., the free end and fixed end at $\Gamma=4$ and $\Gamma=5$. Generally, it is known that an odd number of cells are produced when the boundary conditions is a free end with Non-Newtonian fluid. In the case of the fixed end, we observed that both even number and odd number of cells were produced.

The Taylor vortexes that occurred are illustrated in easy-to-understand manner in Figure 2 and Figure 3. These figures represent cross sections when the Newtonian fluid produced the Taylor vortex in the space between inner and outer cylinders. The figures marked 1, 2, 3, and 4 correspond to the Taylor vortexes with three, four, five and six cells, respectively.

Figure 4 and Figure 5 display the results for $\Gamma=4$. The vertical axis represents the acceleration time, and the horizontal axis corresponds to the Reynolds number. As can be seen from these figures, the non-uniqueness was confirmed at when the upper end wall is free edge, because each mode and cell appear. In the meantime, the vortex flow changes to the anomalous modes for acceleration time $5 \mathrm{~s}, 6 \mathrm{~s}$, and $7 \mathrm{~s}$ at the Reynolds number of 600 , and for acceleration time $2 \mathrm{~s}$ at the Reynolds number of 900 . When the upper end wall is fixed, only normal mode of 4 cells appear, which shows the stable state.

Figure 6 and Figure 7 show the results of the fixed end at $\Gamma=5$. The vertical axis represents the acceleration time, and the horizontal axis corresponds to the Reynolds number. As can be seen from these figures, only normal mode for five cells is observed when the upper end wall is fixed, which shows the stable state. The non-uniqueness can be confirmed when the upper end wall is fixed, because each mode and cell appear. In the meantime, the vortex flow changes to the anomalous mode for acceleration time $2 \mathrm{~s}$ and $3 \mathrm{~s}$ at the Reynolds number of 700 , and for acceleration time $2 \mathrm{~s}$ at the Reynolds number of 1000 . When the upper end wall is free edge, only normal mode of 5 cells appear, which shows the stable state.

\subsection{Numerical Results}

In the case of a free end, an odd number of cells in which the flow rotates clockwise are normal cells at both lower and upper ends. In the other cases, all the cells become anomalous cells. The kinds of the cells obtained in the present analysis are shown in Figure 8. In the figures, the left hand side and right hand side indicate the inner cylinder and the outer cylinder, respectively. Cold colored area indicates the vortex swirling in the counter-clockwise direction, and ward colored area shows the vortex swirling in the clockwise direction. From left to right, the figures correspond to 3, 4 5, 6 and 7 cells. As a result of the analysis at the aspect ratio $4.0,3,4,5$, and 6 cells appeared. The analysis at the aspect ratio 5.0 revealed that 5,6 , and 7 cells appeared. 
Figure 9 represents the table of the experimental results obtained at the aspect ratios of 4.0 and 5.0 while changing the acceleration time at each Reynolds number. The vertical axis represents the respective Reynolds number, and the horizontal axis corresponds to the acceleration time. From these results, it is seen that the number of cells at the aspect ratios of 4.0 becomes close to five with the increase in the Reynolds number and the acceleration time. For the aspect ratio 5.0, it was observed that the number of cells tends to become close to five at the Reynold number of 100, 800, 900, and 1000. For the Reynold number of 200, six cells appeared with the increase in the acceleration time. In the other cases, number of cells becomes nearly seven. Also, it was found that there are more changes in the number of cells at the aspect ratio of 4.0 than that of 5.0.

Figure 10 shows the flow mode obtained when the both end walls are fixed. These are 2 cells, 4 cells, 6 cells, and anomalous 4 cells, from the left. When an even number of cells are produced, such that fluid rotates clockwise at the lower end while it rotates counterclockwise at the higher end, the cells become normal cell. All other cases are called anomalous cells. At the aspect ratio 4, four kinds of cells (two cells, four cells, six cells, and variation cells) appeared. It is observed that the number of cells tends to easily become 4 cells, 6 cells, and 4 cells at the Reynolds numbers of 100 - 300, 400 - 500, and 700 - 1000, respectively. At the Reynolds number of 900 and 1000, the anomalous cells appear at the acceleration time of $1 \mathrm{sec}$. Two kinds of cells ( 4 cells and 6 cells) appeared at the aspect ratio 5. At the Reynolds number of 100 , it was seen that the 4 cells tend to appear as the acceleration time becomes slow. In other cases, the number of cells was approximately six. Figure 11 shows the graph where the vertical axis represents the Reynolds number and horizontal axis represents the acceleration time.
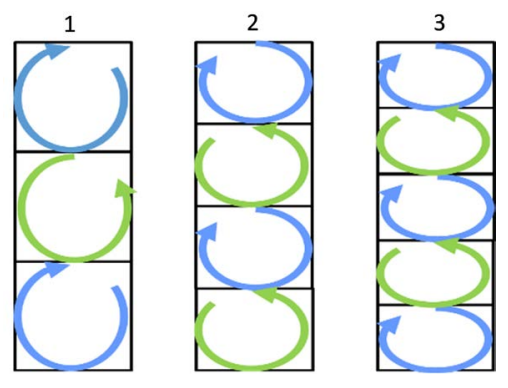

Figure 2. Flow mode when upper end wall is free edge.

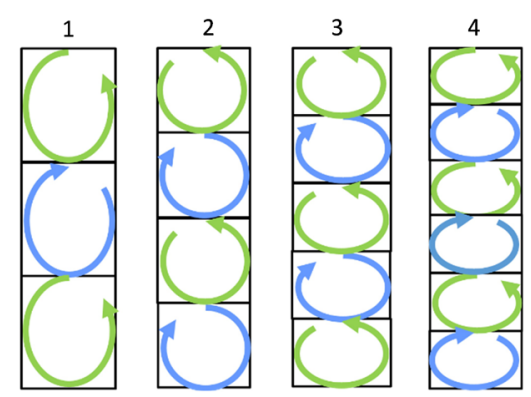

Figure 3. Flow mode when upper end wall is fixed edge. 


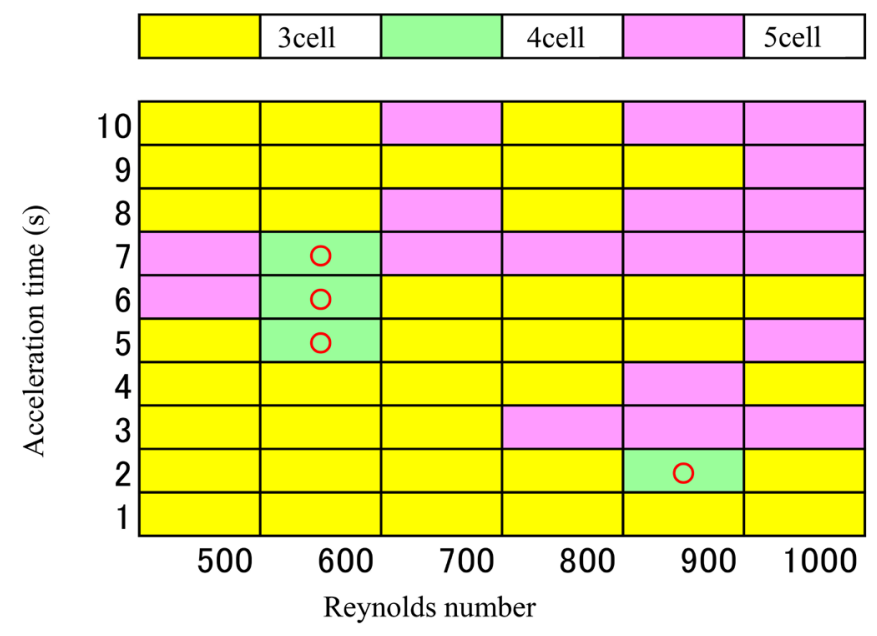

Figure 4. Graph of quantity of the acceleration time and the Reynolds number ( $\Gamma=4$ free edge).

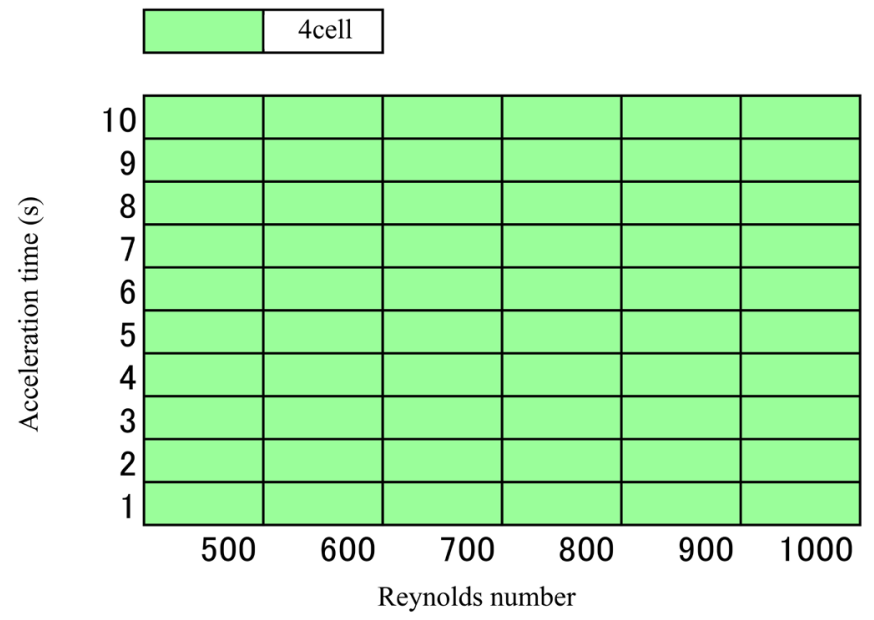

Figure 5. Graph of quantity of the acceleration time and the Reynolds number ( $\Gamma=4$ fixed edge).

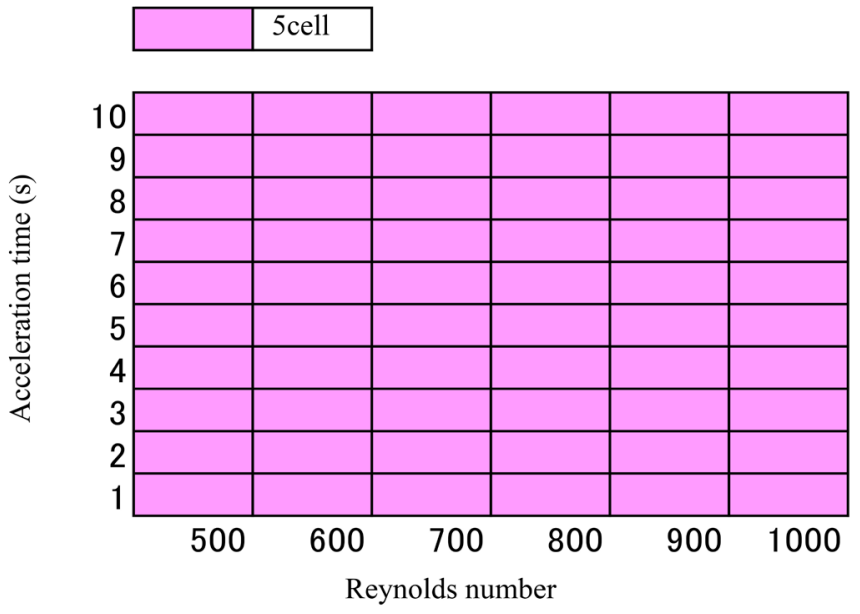

Figure 6. Graph of quantity of the acceleration time and the Reynolds number ( $\Gamma=5$ free edge). 


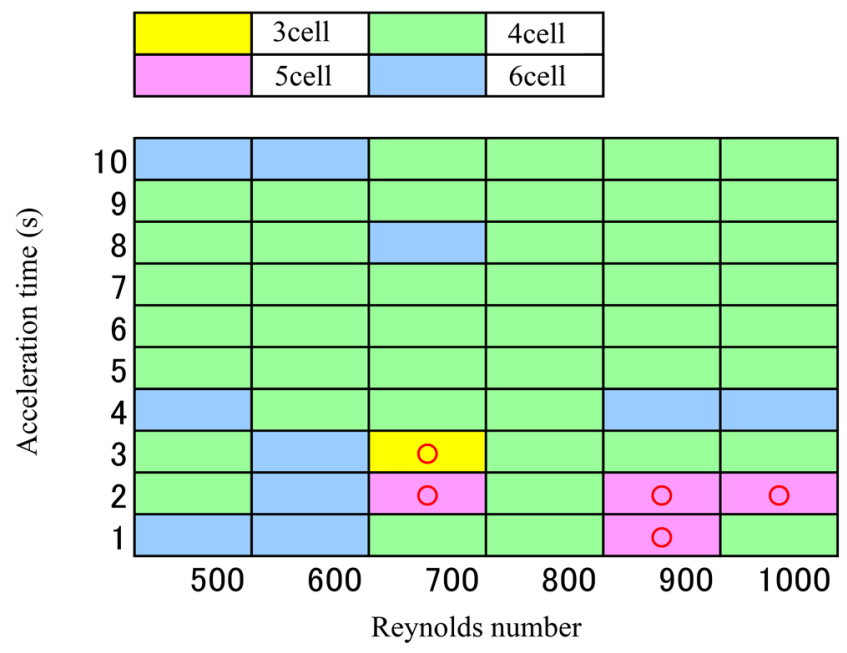

Figure 7. Graph of quantity of the acceleration time and the Reynolds number ( $\Gamma=5$ fixed edge).
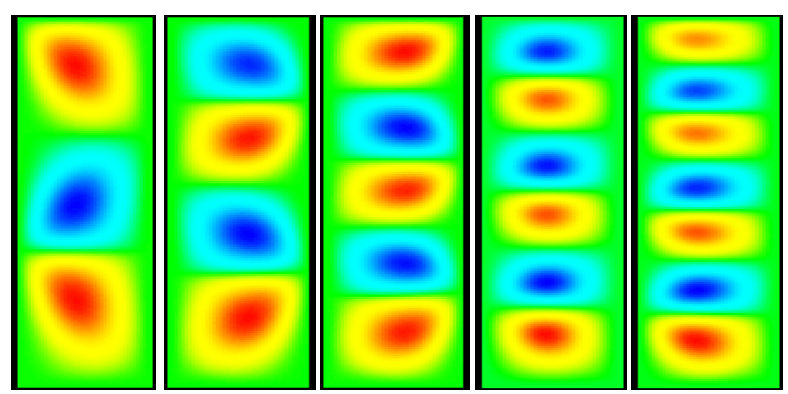

Figure 8. Flow mode of free end wall vortex flow.

\begin{tabular}{|c|c|c|c|c|c|c|c|c|c|c|c|}
\hline \multirow{11}{*}{4} & 10 & 4 & 5 & 5 & 5 & 5 & 5 & 5 & $\overline{4}$ & 4 & 5 \\
\hline & 9 & 4 & 5 & 5 & 5 & 5 & 5 & 4 & 4 & 5 & 5 \\
\hline & 8 & 4 & 5 & 5 & 5 & 6 & 6 & 4 & 5 & 5 & 5 \\
\hline & 7 & 4 & 5 & 5 & 5 & 6 & 6 & 5 & 5 & 5 & 5 \\
\hline & 6 & 4 & 5 & 5 & 6 & 6 & 5 & 5 & 5 & 3 & 5 \\
\hline & 5 & 4 & 5 & 5 & 6 & 5 & 5 & 5 & 5 & 5 & 5 \\
\hline & 4 & 4 & 5 & 6 & 5 & 5 & 5 & 5 & 5 & 5 & 5 \\
\hline & 3 & 4 & 5 & 4 & 5 & 5 & 5 & 5 & 5 & 5 & 3 \\
\hline & 2 & 4 & 4 & 5 & 5 & 5 & 5 & 5 & 5 & 4 & 5 \\
\hline & 1 & 4 & 3 & 5 & 5 & 5 & 3 & 5 & 5 & 5 & 5 \\
\hline & & 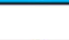 & & & & & & & - & 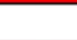 & \\
\hline \multirow{11}{*}{5} & 10 & 5 & 6 & 6 & 7 & 7 & 7 & 7 & 7 & 5 & 5 \\
\hline & 9 & 5 & 6 & 6 & 7 & 7 & 7 & 7 & 5 & 5 & 5 \\
\hline & 8 & 5 & 6 & 7 & 7 & 7 & 7 & 7 & 5 & 5 & 5 \\
\hline & 7 & 5 & 6 & 7 & 7 & 7 & 7 & 7 & 5 & 5 & 5 \\
\hline & 6 & 5 & 6 & 7 & 7 & 7 & 7 & 7 & 5 & 5 & 5 \\
\hline & 5 & 5 & 6 & 7 & 7 & 7 & 7 & 7 & 5 & 5 & 5 \\
\hline & 4 & 5 & 6 & 7 & 7 & 7 & 7 & 7 & 5 & 6 & 5 \\
\hline & 3 & 5 & 5 & 7 & 7 & 7 & 7 & 6 & 5 & 5 & 5 \\
\hline & 2 & 5 & 5 & 7 & 7 & 6 & 5 & 5 & 5 & 5 & 5 \\
\hline & 1 & 5 & 7 & 7 & 6 & 6 & 7 & 7 & 7 & 5 & 5 \\
\hline & & 100 & 200 & 300 & 400 & 500 & 600 & 700 & 800 & 900 & 1000 \\
\hline $\begin{array}{l}\text { Aspect } \\
\text { ratio }\end{array}$ & $\begin{array}{l}\text { Accel. } \\
\text { time }\end{array}$ & \multicolumn{10}{|c|}{ Reynolds number } \\
\hline
\end{tabular}

Figure 9. Graph of the Reynolds number and acceleration time (Free end wall). 


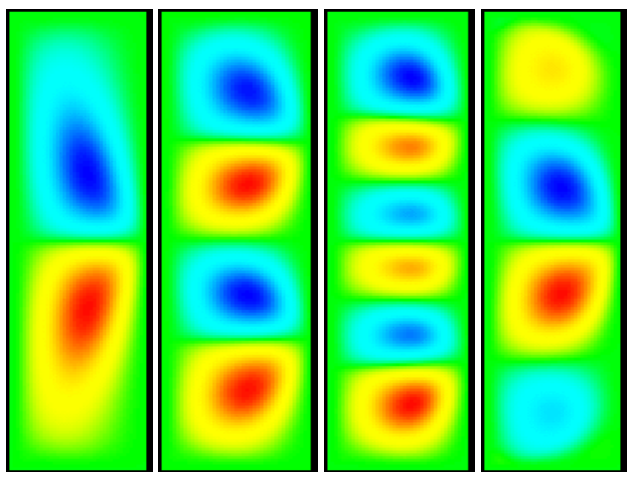

Figure 10. Flow mode of fixed end wall vortex flow.

\begin{tabular}{|c|c|c|c|c|c|c|c|c|c|c|c|}
\hline & 10 & 4 & 4 & 4 & 4 & 6 & 4 & 4 & 4 & 4 & 4 \\
\hline \multirow{9}{*}{4} & 9 & 4 & 4 & 4 & 6 & 6 & 4 & 4 & 4 & 4 & 4 \\
\hline & 8 & 4 & 4 & 4 & 6 & 6 & 4 & 4 & 4 & 4 & 2 \\
\hline & 7 & 4 & 4 & 4 & 6 & 6 & 4 & 2 & 4 & 4 & 2 \\
\hline & 6 & 4 & 4 & 4 & 6 & 6 & 4 & 4 & 4 & 4 & 4 \\
\hline & 5 & 4 & 4 & 4 & 6 & 6 & 4 & 4 & 4 & 2 & 4 \\
\hline & 4 & 4 & 2 & 4 & 6 & 6 & 4 & 4 & 4 & 4 & 2 \\
\hline & 3 & 4 & 2 & 4 & 6 & 6 & 4 & 4 & 4 & 4 & 4 \\
\hline & 2 & 4 & 2 & 4 & 6 & 6 & 4 & 4 & 4 & 4 & 2 \\
\hline & 1 & 4 & 2 & 4 & 6 & 6 & 2 & 4 & 4 & $\mathrm{~A} 4$ & $\mathrm{~A} 4$ \\
\hline & & & & & & & & & & & \\
\hline \multirow{11}{*}{5} & 10 & 4 & 6 & 6 & 6 & 6 & 6 & 6 & 6 & 6 & 6 \\
\hline & 9 & 4 & 6 & 6 & 6 & 6 & 6 & 6 & 6 & 6 & 6 \\
\hline & 8 & 4 & 6 & 6 & 6 & 6 & 6 & 6 & 6 & 6 & 6 \\
\hline & 7 & 4 & 6 & 6 & 6 & 6 & 6 & 6 & 6 & 6 & 6 \\
\hline & 6 & 4 & 6 & 6 & 6 & 6 & 6 & 6 & 6 & 6 & 6 \\
\hline & 5 & 4 & 6 & 6 & 6 & 6 & 6 & 6 & 6 & 6 & 6 \\
\hline & 4 & 4 & 6 & 6 & 6 & 6 & 6 & 6 & 6 & 6 & 4 \\
\hline & 3 & 6 & 6 & 6 & 6 & 6 & 6 & 4 & 6 & 6 & 6 \\
\hline & 2 & 6 & 6 & 6 & 6 & 6 & 6 & 6 & 6 & 6 & 6 \\
\hline & 1 & 6 & 6 & 6 & 6 & 6 & 4 & 6 & 6 & 6 & 6 \\
\hline & & 100 & 200 & 300 & 400 & 500 & 600 & 700 & 800 & 900 & 1000 \\
\hline $\begin{array}{l}\text { Aspect } \\
\text { ratio }\end{array}$ & $\begin{array}{l}\text { Accel. } \\
\text { time }\end{array}$ & \multicolumn{10}{|c|}{ Reynolds number } \\
\hline
\end{tabular}

Figure 11. Graph of the Reynolds number and acceleration time (Fixed end wall).

\section{Conclusion}

In the present study, the acceleration time of the inner cylinder was changed. In this experiments, we have analyzed how the Taylor vortex flow, produced between two coaxial rotating cylinders sharing the central axis, changes with the aspect ratio, the Reynolds number and the acceleration time for the both free and fixed ends. Non-uniqueness of Taylor vortex flow is investigated both experimentally and numerically. Both results show good agreement qualitatively.

\section{Acknowledgements}

We thank Prof. Takashi Watanabe, Nagoya University who provided insight and expertise that greatly assisted the research. We would also like to show our gratitude to members in our laboratory for sharing their pearls of wisdom with us during the course of this research, and we thank "anonymous" reviewers for 
helpful comments that greatly improved the manuscript.

\section{Conflicts of Interest}

The authors declare no conflicts of interest regarding the publication of this paper.

\section{References}

[1] Taylor, G.I. (1923) VIII. Stability of a Viscous Liquid Contained between Two Rotating Cylinders. Philosophical Transactions of the Royal Society A, 223, 289-343. https://doi.org/10.1098/rsta.1923.0008

[2] Coles, D. (1965) Transition in Circular Couette Flow. Journal of Fluid Mechanics, 21, 385-425. https://doi.org/10.1017/S0022112065000241

[3] Martínez-Arias, B., Peixinho, J., Crumeyrolle, O. and Mutabazi, I. (2014) Effect of the Number of Vortices on the Torque Scaling in Taylor-Couette Flow. Journal of Fluid Mechanics, 748, 756-767. https://doi.org/10.1017/jfm.2014.213

[4] Ostilla-Mónico, R., Lohse, D. and Verzicco, R. (2016) Effect of Roll Number on the Statistics of Turbulent Taylor-Couette Flow. Physical Review Fluids, 1, Article ID: 054402. https://doi.org/10.1103/PhysRevFluids.1.054402

[5] Xia, Z.H., et al. (2018) Multiple States in Turbulent Plane Couette Flow with Spanwiserotation. Journal of Fluid Mechanics, 837, 477-490. https://doi.org/10.1017/jfm.2017.869

[6] Benjamin, T.B. and Mullin, T. (1981) Anomalous Mode in the Taylor Experiment. Proceedings of the Royal Society of London. Series A, 377, 221-249. https://doi.org/10.1098/rspa.1981.0122

[7] Alziary de Roquefort, T. and Grillaud, G. (1978) Computation of Taylor Vortex Flow by a Transient Implicit Method. Computers \& Fluids, 6, 259-269. https://doi.org/10.1016/0045-7930(78)90017-8

[8] Furukawa, H., Hanaki, M. and Watanabe T. (2008) Influence of Initial Flow on Taylor Vortex Flow. Journal of Fluid Science and Technology, 3, 129-136. https://doi.org/10.1299/jfst.3.129

[9] Furukawa, H. (2012) Interactive Visualization System of Taylor Vortex Flow Using Stokes' Stream Function. World Journal of Mechanics, 2, 188-196.

https://doi.org/10.4236/wjm.2012.24023 